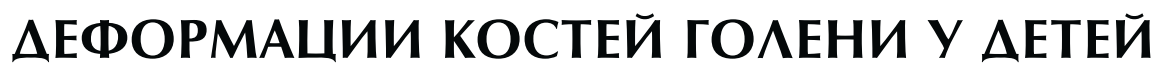 ВСАЕАСТВИЕ ПОВРЕЖАЕНИЯ ЗОНЫ РОСТА: АНААИЗ ХИРУРГИЧЕСКОГО АЕЧЕНИЯ 28 ПАЦИЕНТОВ (ПРЕАВАРИТЕАЬНОЕ СООБЩЕНИЕ)
}

\author{
() В.А. Виленский, А.А. Поздеев, Т.Ф. Зубаиров, Е.А. Захарьян \\ ФГБУ «НИДОИ им. Г.И. Турнера» Минздрава России, Санкт-Петербург
}

Статья поступила в редакцию: 05.10.2017

Статья принята к печати: 24.11.2017

\begin{abstract}
Цель работы - ретроспективно проанализировать результаты двух методов лечения детей с деформациями голени, являющимися следствием парциального синостоза зоны роста.

Материалы и методы. Группу I составили 15 пациентов, которым выполняли остеотомию с одномоментной гиперкоррекцией деформации и чрескостный остеосинтез аппаратом Илизарова с последующим дозированным удлинением сегмента. Группу II составили 13 пациентов, которым выполняли гемиэпифизиодез функционирующей порции поврежденной зоны роста, остеотомию, чрескостный остеосинтез аппаратом Орто-СУВ с последующей коррекцией деформации и удлинением сегмента во времени.

Результаты. В группе I выявлено, что при коррекции варусных деформаций гиперкоррекция по девиации механической оси (ДМО) составила 18,28 \pm 5,25 мм, гиперкоррекция по мМПрББУ (механическому медиальному проксимальному большеберцовому углу) $-14,86 \pm 4,45^{\circ}$; по мЛДББУ (механическому латеральному большеберцовому углу) $-12,85 \pm 3,02^{\circ}$. При коррекции вальгусных деформаций гиперкоррекция по ДМО составила $15,12 \pm 8,28$ мм, гиперкоррекция по мМПрББУ $-10,38 \pm 2,77^{\circ}$; по мЛДББУ $-7,5 \pm 3,9^{\circ}$. В 11 случаях (73\%) отмечался рецидив деформации. При этом минимальные сроки рецидива деформации составили 5 месяцев, максимальные - 16 месяцев.

В группе II точность коррекции (ТК) варусных деформаций по ДМО составила 98 \%, по мМПрББУ и мЛДББУ 94 \%; для вальгусных деформаций по ДМО - 90 \%, по мМПрББУ и мЛДББУ $-96 \%$. ТК антекурвационных деформаций по анатомическому заднему проксимальному большеберцовому углу (аЗПББУ) и анатомическому переднему дистальному большеберцовому углу (аПДББУ) составила $96 \%$, рекурвационных $-92 \%$. Только в одном случае через 6 месяцев после демонтажа аппарата отмечался рецидив деформации. В 2 случаях по мере роста ребенка потребовалось повторное оперативное вмешательство, направленное на устранение неравенства длин конечностей. Заключение. Использование методики эпифизиодеза неповрежденной порции зоны роста в сочетании с остеотомией и чрескостным остеосинтезом на базе компьютерной навигации с последующими дозированными коррекцией деформации и удлинением достоверно снижает частоту рецидивов у пациентов с деформациями голени на фоне физарных синостозов.
\end{abstract}

Ключевые слова: эпифизиодез; компьютерная навигация; гексапод; аппарат Орто-СУВ; коррекция деформации; гиперкоррекция.

\section{TREATMENT OF PEDIATRIC PATIENTS WITH LOWER LEG DEFORMITIES ASSOCIATED WITH PHYSEAL ARREST: ANALYSIS OF 28 CASES}

\author{
(C) V.A. Vilenskiy, A.A. Pozdeev, T.F. Zubairov, E.A. Zakharyan
}

The Turner Scientific Research Institute for Children's Orthopedics, Saint Petersburg, Russia

For citation: Pediatric Traumatology, Orthopaedics and Reconstructive Surgery. 2017;5(4):38-47

Received: 05.10 .2017

Accepted: 24.11.2017

Aim. To retrospectively analyze the results of two treatment methods for lower leg deformities associated with partial growth arrest. 
Materials and methods. Group I comprised 15 children who underwent osteotomy, acute overcorrection, and external fixation by Ilizarov with subsequent lengthening of the segment. Group II comprised 13 patients who underwent epiphysiodesis of the healthy part of the growth plate by drilling, osteotomy with external fixation by use of an Ortho-SUV Frame, and subsequent gradual deformity correction and lengthening.

Results. In group I, overcorrection of varus deformities by mechanical axis deviation (MAD) was $18.28 \pm 5.25 \mathrm{~mm}$, overcorrection by mechanical medial proximal tibial angle (mMPTA) was $14.86 \pm 4.45^{\circ}$, and overcorrection by mechanical lateral distal tibial angle (mLDTA) was $12.85 \pm 3.02^{\circ}$. Overcorrection of valgus deformities according to MAD was $15.12 \pm 8.28 \mathrm{~mm}$, overcorrection by mMPTA was $10.38 \pm 2.77^{\circ}$, and overcorrection by mLDTA was $7.5 \pm 3.9^{\circ}$. Recurrence of the deformity was observed in 11 (73\%) cases (range, 5-16 months).

In group II, the accuracy of correction (AC) in varus deformities for MAD was $98 \%$ and $94 \%$ for $\mathrm{mMPTA}$ and mLDTA. For valgus deformities, AC for MAD was $90 \%$ and $96 \%$ for mMPTA and mLDTA. The AC for anatomical proximal posterior tibial angle and anatomical anterior distal tibial angle was $96 \%$ for procurvation deformities and that for recurvation deformities was 92\%. Deformity recurrence was observed in only one case within 6 months after frame removal. In 2 cases, repeat limb length discrepancy correction surgeries were performed.

Conclusion. Use of epiphysiodesis of the healthy portion of the growth plate in combination with osteotomy, computerassisted external fixation with subsequent gradual deformity correction, and lengthening in patients with deformities associated with partial physeal arrest significantly decreased the number of deformity recurrences.

Keywords: epiphysiodesis; deformity correction; hexapods; software-based external fixation; six-axis frames; Ortho-SUV Frame.

\section{Введение}

Отличительной особенностью детского скелета является наличие функционирующих зон роста. Повреждение зоны роста в результате травмы или патологического процесса (опухоль, инфекция, дистрофия) зачастую приводит к формированию парциального физарного синостоза. По мере роста ребенка здоровая часть зоны роста продолжает функционировать, в то время как синостозированная не работает, что ведет к формированию деформации и/или укорочения сегмента [1-3].

Доказано, что наличие осевых деформаций голени вызывает биомеханические нарушения, лежащие в основе формирования остеоартроза коленного [4], голеностопного и подтаранного суставов $[5,6]$. Таким образом, необходимость выполнения оперативного лечения детей с деформациями голени не вызывает сомнения.

В ортопедии детского возраста существует несколько подходов к оперативному лечению деформаций голени, являющихся следствием повреждения зоны роста. Так, ряд авторов пропагандирует выполнение резекции синостозированной порции зоны роста с заполнением дефекта материалом, препятствующим рецидиву синостоза (жиром, воском, метилметакрилатом) [7]. Также комбинируют данную процедуру с корригирующей остеотомией [8]. Указанный метод имеет ограничения: угловая деформация не должна превышать 10 градусов; площадь синостоза не должна превышать $50 \%$ от площади зоны роста; необходим активный рост ребенка; при наличии значимого укорочения неэффективен, так как длина сегмента не восстанавливается [7]. Также отмечается высокая частота рецидивов синостозов [8].
Стандартом лечения деформаций, сопровождающихся укорочением, является остеотомия сегмента в сочетании с чрескостным остеосинтезом. Данный метод позволяет устранить деформацию с одновременным удлинением сегмента дозированно, во времени, путем формирования дистракционного регенерата [9-11]. Однако остеотомия в сочетании с чрескостным остеосинтезом не гарантируют положительного результата лечения у детей. При отсутствии вмешательства на зоне роста дальнейший рост ребенка закономерно приводит к рецидиву деформации. Это побудило ряд ортопедов в случаях деформаций, сопровождающихся физарным синостозом, выполнять гиперкоррекцию [12] с целью отдаления во времени рецидива деформации. Величина гиперкоррекции, как и ее целесообразность, не доказана.

В единичных публикациях упоминается о положительном эффекте от сочетания остеотомии поврежденного сегмента, эпифизиодеза неповрежденной порции зоны роста путем рассверливания и чрескостного остеосинтеза [13]. При этом производят не только коррекцию деформации, но и переудлинение поврежденного сегмента. Величину переудлинения рассчитывают с использованием методик прогнозирования [14]. Однако в литературе мы не нашли достоверных данных об эффективности этой методики. В качестве чрескостного аппарата целесообразно использование гексаподов - устройств на базе компьютерной навигации, обладающих высокой точностью коррекции деформаций [15-18].

Цель данной работы - ретроспективно проанализировать результаты двух методов оператив- 
ного лечения детей с деформациями голени, являющимися следствием парциального синостоза зоны роста.

\section{Материалы и методы}

Анализу подлежали результаты лечения 28 пациентов, пролеченных в период с 2009 по 2015 г. Обязательным условием было подписание информированного согласия родителями ребенка (или опекунами) на участие в клиническом исследовании и проведение операции. У всех пациентов имели место деформации костей голени, ассоциированные с укорочением поврежденного сегмента. Основным условием включения пациента в исследование было наличие деформации голени с рентгенологическими признаками парциального синостоза зоны роста.

Группу I составили 15 пациентов, которым выполняли остеотомию костей голени в сочетании с чрескостным остеосинтезом. При этом хирургического вмешательства на зоне роста не производили. Коррекцию деформации осуществляли одномоментно или дозированно методами чрескостного остеосинтеза. При этом голени придавали положение гиперкоррекции по углу и по длине.

Группу II составили 13 пациентов, которым выполняли эпифизиодез неповрежденной порции зоны роста путем ее рассверливания под контролем электронно-оптического преобразователя, остеотомии костей голени и чрескостный остеосинтез. Для коррекции деформации использовался узел Орто-СУВ. При этом целью было восстановление нормальных значений референтных линий и углов (РЛУ) [19] и переудлинение сегмента. Величину переудлинения рассчитывали при помощи программы PaleyGrowth.

В группе I было 6 мальчиков и 9 девочек, средний возраст исследуемых на момент начала лечения составлял $11,0 \pm 2,6$ года (минимальный -6 , максимальный -15 лет). В 4 случаях причиной деформации была травма, в 5 - болезнь Блаунта, в 6 - перенесенный в младенчестве гематогенный остеомиелит (ПОГО). В 10 случаях деформация сформировалась вследствие повреждения проксимальной зоны роста, в 5 случаях - дистальной. В 8 случаях деформация была сложной (СД), в 5 случаях - средней степени тяжести (ССС) и в $2-$ простой (ПД) согласно классификации деформаций длинных костей [20].

В группе II было 7 девочек и 6 мальчиков, средний возраст исследуемых на момент начала лечения составлял $10,6 \pm 2,9$ года (минимальный -7 , максимальный -14 лет). В 7 случаях причиной деформации была травма, в $4-$ болезнь Блаунта, в 2 - ПОГО. В 7 случаях деформация сформировалась вследствие повреждения проксимальной зоны роста, в 6 случаях - дистальной. В 6 случаях имели место СД, в 6 случаях - ССС и в 1 случае - ПД.

В группе I оценивали величину удлинения, величину переудлинения, период коррекции деформации (ПК), величину гиперкоррекции, индекс внешней фиксации (ИВФ), количество и характер осложнений. Величину гиперкоррекции оценивали, сравнивая полученные в результате коррекции значения РЛУ с крайним значением из диапазона нормальных.

В группе II оценивали величину удлинения, величину переудлинения, ПК, точность коррекции, ИВФ, количество и характер осложнений. При анализе точности коррекции оценивали показатели РЛУ.

\section{Результаты}

Основные результаты представлены в таблицах 1 и 3.

\section{Группа I}

При коррекции варусных деформаций гиперкоррекция по девиации механической оси (ДМО) составила 18,28 $\pm 5,25$ мм, гиперкоррекция по мМПрББУ (механическому медиальному проксимальному большеберцовому углу) $-14,86 \pm 4,45^{\circ}$; по мЛДББУ (механическому латеральному большеберцовому углу) $-12,85 \pm 3,02^{\circ}$. При коррекции вальгусных деформаций гиперкоррекция по ДМО составила $15,12 \pm 8,28$ мм, гиперкоррекция по мМПрББУ $-10,38 \pm 2,77^{\circ}$; по мЛДББУ $7,5 \pm 3,9^{\circ}$. В 11 случаях (73\%) отмечался рецидив деформации. При этом минимальные сроки рецидива деформации составили 5 месяцев, максимальные - 16 месяцев. В 12 случаях было осуществлено переудлинение сегмента. В 3 случаях переудлинение преднамеренно не выполнялось. Это связано с тем, что предоперационное укорочение сегмента составляло более $6 \mathrm{~cm}$. Следует отметить, что период коррекции деформации, приведенный в таблице 1 для группы I, подсчитан только у пяти пациентов, которым коррекция выполнялась дозированно, при помощи унифицированных узлов РНЦ ВТО. Во всех остальных случаях коррекция деформации была одномоментной и осуществлялась интраоперационно.

Мы наблюдали следующие осложнения. Во всех случаях в ходе удлинения и коррекции деформации отмечались воспалительные изменения в области мест выходов одного или более чрескостных элементов. При этом антибактериальная терапия для купирования воспалительного 
Рефрерентные кинии и углы в группе I

\begin{tabular}{|c|c|c|c|}
\hline Показатель & Нормальные значения & До операции & После коррекции \\
\hline \multicolumn{4}{|c|}{ Фронтальная плоскость } \\
\hline \multicolumn{4}{|c|}{ Bapyc } \\
\hline ДМО & $0 \pm 9,7$ мм кнутри & $36,8 \pm 9,9$ мм кнутри & $18,3 \pm 7,7$ мм кнаружи \\
\hline мМПрББУ & $85-90^{\circ}$ & $76 \pm 6,7^{\circ}$ & $99,8 \pm 4,4^{\circ}$ \\
\hline мЛДББУ & $86-92^{\circ}$ & $100,7 \pm 5^{\circ}$ & $79,1 \pm 3^{\circ}$ \\
\hline \multicolumn{4}{|c|}{ Вальгус } \\
\hline ДМО & $0 \pm 9,7$ мм кнутри & $31,8 \pm 8,9$ мм кнаружи & $15,2 \pm 8,3$ мм кнутри \\
\hline мМПрББУ & $85-90^{\circ}$ & $100,1 \pm 6,5^{\circ}$ & $79,6 \pm 2,7^{\circ}$ \\
\hline мЛДББУ & $86-92^{\circ}$ & $74,2 \pm 6,4^{\circ}$ & $97,5 \pm 3,9^{\circ}$ \\
\hline \multicolumn{4}{|c|}{ Сагиттальная плоскость } \\
\hline \multicolumn{4}{|c|}{ Рекурвация } \\
\hline аЗПББУ & $77-84^{\circ}$ & $95,1 \pm 5,1^{\circ}$ & $82,7 \pm 3,9^{\circ}$ \\
\hline аПДББУ & $78-82^{\circ}$ & $70,4 \pm 3,9^{\circ}$ & $83,6 \pm 5,6^{\circ}$ \\
\hline \multicolumn{4}{|c|}{ Антекурвация } \\
\hline аЗПББу & $77-84^{\circ}$ & $68,3 \pm 6,2^{\circ}$ & $86,1 \pm 5,5^{\circ}$ \\
\hline аПДББУ & $78-82^{\circ}$ & $88,7 \pm 6,5^{\circ}$ & $75,7 \pm 2,7^{\circ}$ \\
\hline
\end{tabular}

Примечание: мМПрББУ - механический медиальный проксимальный угол большеберцовой кости; мЛДББУ - механический латеральный дистальный угол большеберцовой кости; ДМО - девиация механической оси; аЗПББУ - анатомический задний проксимальный большеберцовый угол; аПДББУ - анатомический передний дистальный большеберцовый угол.

Таблица 2

Редерентные минии и углы в группе II

\begin{tabular}{|c|c|c|c|}
\hline Показатель & Нормальные значения & До операции & После коррекции \\
\hline \multicolumn{4}{|c|}{ Фронтальная плоскость } \\
\hline \multicolumn{4}{|c|}{ Bapyc } \\
\hline ДМО & $0 \pm 9,7$ мм кнутри & $27,4 \pm 6,7$ мм кнутри & $2,5 \pm 8,8$ мм кнутри \\
\hline мМПрББУ & $85-90^{\circ}$ & $72,8 \pm 8,5^{\circ}$ & $86,2 \pm 3,8^{\circ}$ \\
\hline мЛДББУ & $86-92^{\circ}$ & $102,7 \pm 8,4^{\circ}$ & $87,6 \pm 4,5^{\circ}$ \\
\hline \multicolumn{4}{|c|}{ Вальгус } \\
\hline ДМО & $0 \pm 9,7$ мм кнутри & $44,3 \pm 10,2$ мм кнаружи & $0,6 \pm 5,2$ мм кнутри \\
\hline мМПрББУ & $85-90^{\circ}$ & $104,5 \pm 8,5^{\circ}$ & $89,5 \pm 6,8^{\circ}$ \\
\hline мЛДББУ & $86-92^{\circ}$ & $74,2 \pm 6,4^{\circ}$ & $97,5 \pm 3,9^{\circ}$ \\
\hline \multicolumn{4}{|c|}{ Сагиттальная плоскость } \\
\hline \multicolumn{4}{|c|}{ Рекурвация } \\
\hline аЗПББУ & $77-84^{\circ}$ & $95,1 \pm 5,1^{\circ}$ & $82,7 \pm 3,9^{\circ}$ \\
\hline аПДББу & $78-82^{\circ}$ & $70,4 \pm 3,9^{\circ}$ & $83,6 \pm 5,6^{\circ}$ \\
\hline \multicolumn{4}{|c|}{ Антекурвация } \\
\hline аЗПББУ & $77-84^{\circ}$ & $68,3 \pm 6,2^{\circ}$ & $86,1 \pm 5,5^{\circ}$ \\
\hline аПДББу & $78-82^{\circ}$ & $88,7 \pm 6,5^{\circ}$ & $75,7 \pm 2,7^{\circ}$ \\
\hline
\end{tabular}

Примечание: мМПрББУ - механический медиальный проксимальный угол большеберцовой кости; мЛДББУ - механический латеральный дистальный угол большеберцовой кости; ДМО - девиация механической оси; аЗПББУ - анатомический задний проксимальный большеберцовый угол; аПДББУ - анатомический передний дистальный большеберцовый угол. 
Результаты, полученные в группах I и II

\begin{tabular}{|l|c|c|}
\hline \multicolumn{1}{|c|}{ Показатель } & Группа I & Группа II \\
\hline Удлинение, мм & $46,4 \pm 7,2$ & $54,1 \pm 9,3$ \\
\hline Переудлинение, мм & $18,6 \pm 6,2$ & $22,4 \pm 8,2$ \\
\hline Время дистракции, дней & $48,3 \pm 7,3$ & $46,3 \pm 12,2$ \\
\hline Период коррекции, дней & $12,4 \pm 6,1$ & $14,4 \pm 4,2$ \\
\hline Индекс внешней фиксации, дней/см & $34,4 \pm 8,2$ & $28,3 \pm 10,1$ \\
\hline
\end{tabular}

процесса применялась только у трех пациентов. У 4 пациентов в ходе дистракции возникла контрактура голеностопного сустава, у 3 - коленного. Ни одно из данных осложнений не потребовало оперативного лечения. В 1 случае отмечалось формирование ложного сустава, вследствие чего были произведены пластика аутокостью и повторная внешняя фиксация. В 11 случаях (73\%) отмечался рецидив деформации. При этом минимальные сроки рецидива деформации составили 5 месяцев, максимальные - 16 месяцев. Во всех этих случаях были выполнены повторные оперативные вмешательства. Все 4 пациента с отсутствием рецидива деформации на момент начала лечения были старше 13 лет.

\section{Группа II}

Основные результаты лечения пациентов группы II представлены в таблицах 2 и 3. Следует отметить, что точность коррекции варусных деформаций по ДМО составила 98 \%, по мМПрББУ и мЛДББУ - $94 \%$; для вальгусных деформаций по ДМО - 90 \%, по мМПрББУ и мЛДББУ - $96 \%$. Точность коррекции антекурвационных деформаций по анатомическому заднему проксимальному большеберцовому углу (аЗПББУ) и анатомическому переднему дистальному большеберцовому углу (аПДББУ) составила $96 \%$, рекурвационных - $92 \%$.

Наблюдались следующие осложнения. Воспалительные изменения мягких тканей в области выходов чрескостных элементов отмечались во всех случаях. В 2 случаях для купирования симптомов поверхностной инфекции потребовалась антибактериальная терапия, в одном случае - удаление чрескостного элемента. В 2 случаях отмечались контрактуры коленного сустава, в 3 случаях - контрактура голеностопного сустава. Во всех случаях физиологическая амплитуда движений в суставе была достигнута при помощи механотерапии в течение периода фиксации и в первый месяц после демонтажа аппарата внешней фиксации. В одном случае через 6 месяцев после демонтажа аппарата возник рецидив де- формации. В 2 случаях по мере роста ребенка потребовалось повторное удлинение поврежденного сегмента.

\section{Обсуждение}

Деформации костей голени у детей, сформировавшиеся в результате повреждения зоны роста травматического или иного генеза, характеризуются высокой частотой рецидивов. Это связано с наличием физарного синостоза, препятствующего равномерному росту ребенка. В случаях раннего выявления и небольшой протяженности синостоза его резекция, по данным разных авторов, может давать хорошие результаты в 40-70 \% случаев [7, 21]. Своевременное выявление синостоза осложняет ряд факторов. При травме с повреждением зоны роста соблюдение всех стандартов лечения, а именно анатомической репозиции и стабильной фиксации, не является гарантией хорошего результата лечения. Тенденция к синостозированию сразу по консолидации перелома не очевидна. Зачастую формирование синостоза может быть определено только при возникновении деформации и укорочения.

При значительной протяженности физарного синостоза у ребенка с продолженным ростом лечение представляется сложным, зачастую «интуитивным» процессом. Ни одно из проанализированных нами руководств не дает единого стандарта лечения. Очевидно, что цель лечения ребенка заключается в устранении деформации, то есть в восстановлении нормальных величин референтных линий и углов, а также в выравнивании длины конечностей. Однако в ряде публикаций звучит термин «гиперкоррекция» $[12,22,23]$, который по сути означает «деформацию в противоположном направлении». Также мы столкнулись с мнением, что гиперкоррекцию следует осуществлять у детей раннего возраста, в то время как у подростков целесообразнее придерживаться нормальных значений референтных линий и углов при коррекции деформации [24]. Ни в одном ис- 
точнике мы не обнаружили информацию о том, какой должна быть величина гиперкоррекции. Это связано с тем, что рост ребенка сложно прогнозировать, а предсказать работу поврежденной зоны роста тем более не просто. Стремление ортопедов к выполнению гиперкоррекции объяснимо нежеланием уничтожать функционирующую порцию зоны роста. Ряд авторов утверждает, что наличие физарного синостоза служит гарантией рецидива деформации вне зависимости от того, выполнена коррекция в соответствии с нормальными значениями РЛУ или с гиперкоррекцией $[13,24]$. Полученные нами результаты не позволяют судить столь однозначно о несостоятельности данного метода, однако $70 \%$ рецидивов деформации в сроки от 5 до 16 месяцев предрасполагают к пересмотру показаний по его использованию. Кроме этого, мы считаем, что в основе гиперкоррекции лежит смещение осевой нагрузки на другой, еще «не скомпрометированный» отдел сустава. Предполагаем, что перегрузка здоровой части сустава, даже на протяжении 5-16 месяцев, не может положительно сказываться на продолжительности «жизни сустава».

Ранее опубликованные Horn et al. результаты лечения 5 пациентов с посттравматическими деформациями нижних конечностей на фоне физарных синостозов свидетельствуют о положительном эффекте от сочетания эпифизиодеза неповрежденной порции зоны роста сверлом в сочетании с остеотомией и чрескостным остеосинтезом [13]. В ряде источников данную методику приводят в качестве рекомендации [17], но мы не смогли найти результатов клинического исследования на достоверной выборке больных. В одном из источников приводятся данные о сочетании корригирующей остеотомии и резекции здоровой порции зоны роста с целью эпифизиодеза при лечении 21 пациента с посттравматическими деформациями длинных костей [25]. Автор утверждает, что резекция сохранившейся порции зоны роста с последующим созданием эпифизиодеза представляет собой рациональный компонент в комплексе оперативного лечения детей с посттравматическими деформациями конечностей, так как предотвращает рецидивирование деформаций и способствует уменьшению кратности оперативных вмешательств на протяжении периода роста ребенка.

Известно, что современные компьютер-ассистированные чрескостные аппараты, так называемые гексаподы, работают на основе расчетов, выполненных в компьютерной программе, и позволяют перемещать костные фрагменты в трех плоскостях и при шести степенях свободы. До- казана высокая точность коррекции деформаций при использовании гексаподов $[15,16,18,26]$. В нашем исследовании мы применяли аппарат Орто-СУВ - единственный на сегодняшний день российский гексапод. Данное устройство, по сути, является не чрескостным аппаратом, а репозиционным узлом на базе компьютерной навигации, который можно использовать с внешними опорами практически всех циркулярных аппаратов $[18,26]$. Однако на сегодняшний день сведений о применении данного устройства в детской практике очень мало [27]. Проведенный нами анализ свидетельствует о том, что точность коррекции деформации при помощи узла Орто-СУВ составляет от 90 до $98 \%$ в зависимости от плоскости деформации. Полученные результаты демонстрируют, что коррекция деформации при помощи чрескостного остеосинтеза на базе компьютерной навигации в сочетании с эпифизиодезом зоны роста и переудлинением сегмента дает хорошие результаты. Так, только в одном случае отмечался рецидив деформации. При детальном анализе этого случая выяснилось, что желаемый эпифизиодез несиностозированной порции зоны роста не был достигнут. Вероятно, это связано с непрецизионностью соблюдения техники эпифизиодеза.

Также интересен вопрос: следует ли коррекцию деформации у детей выполнять одномоментно или методами чрескостного остеосинтеза дозированно, во времени? Наши данные говорят о том, что в группе с дозированной коррекцией деформации индекс внешней фиксации был достоверно меньше, чем в группе с одномоментной коррекцией и последующей дистракцией.

Обсуждению подлежит также тема переудлинения сегмента. На сегодняшний день существует несколько методов прогнозирования роста: арифметический метод [28], графический метод Moseley [29], метод Green-Anderson [30], метод Multiplier [31]. В нашей работе мы использовали метод прогнозирования роста Multiplier, который лежит в основе работы компьютерного приложения для iPhone PaleyGrowth. Согласно данным D. Paley et al. указанный метод достаточно точно определяет неравенство длин конечностей по достижении костной зрелости [32]. Величину переудлинения мы рассчитывали, исходя из настоящей длины сегмента и прогнозируемой длины здорового сегмента по достижении им костной зрелости. В 3 случаях мы не смогли выполнить переудлинение на требуемую величину, в связи с тем что она превышала 6 см (величину, которую мы принимаем за максимальную для удлинения в случаях последствий травм и приобретенных заболеваний). В 2 случаях прогнозирование ока- 
залось недостаточно точным, и по достижении ребенком костной зрелости укорочение больного сегмента составило 25 и 30 мм соответственно. Это подтверждает данные Lee et al. [14], которые оценивали точность существующих методов прогнозирования роста и пришли к выводам, что ни один из них не является абсолютно точным. Одному из указанных пациентов выполнено удлинение «больной» голени, а второму - укорачивающая остеотомия здоровой. Недостатком представленного исследования является то, что из 13 пациентов только 7 достигли костной зрелости. Таким образом, мы не можем с уверенностью утверждать, что оставшимся 6 пациентам не потребуется в дальнейшем устранение неравенства длин конечностей.

\section{КАинический пример}

Пациентка Б., 6 лет, госпитализирована в плановом порядке с диагнозом: «Посттравматическая деформация правой голени, укорочение правой нижней конечности». Из анамнеза известно, что травма произошла за 2 года до госпитализации в результате падения с высоты. Лечилась консервативно, выполнялась гипсовая иммобилизация. При поступлении девочка предъявляла жалобы на деформацию голени, укорочение, хромоту. Ранее не оперирована. Нарастание деформации родители ребенка заметили через 4 месяца после травмы.

Клиническое обследование и анализ панорамных рентгенограмм (рис. $1, a, 6)$ свидетельствуют, что у ребенка имеет место сложная варусно-антекурвационная деформация правой голени на фоне парциального синостоза медиальной порции дистальной зоны роста правой голени, превышающего 50 \% ее площади. Данные РЛУ до коррекции были следующие: проксимальный медиальный механический угол большеберцовой кости составил $90^{\circ}$; дистальный латеральный механический угол большеберцовой кости $-116^{\circ}$; анатомический задний проксимальный большеберцовый угол - 79, анатомический передний дистальный большеберцовый угол $-86^{\circ}$. Угол варусной деформации составил $26^{\circ}$, угол рекурвационной деформации - $6^{\circ}$, угол наружной торсии большеберцовой кости (по данным КТ) - 15․ Осуществлено стандартное планирование коррекции деформации (рис. 1, в, г).

Выполнено оперативное лечение в объеме: эпифизиодез наружной порции дистальной зоны роста путем рассверливания, остеотомия костей правой голени в н/3, комбинированный чрескостный остеосинтез (рис. 2, $a, 6$ ). Учитывая высоту остеотомии, голеностопный сустав был зафиксирован. Удлинение осуществлялось по штангам аппарата Илизарова с последующей их заменой на узел Орто-СУВ на период коррекции деформации. Расчет коррекции деформации в программе компьютерной навигации для узла Орто-СУВ приведен на рис. 2, в. При этом выполнена стандартная процедура: на прямой и боковой рентгенограммах при помощи инструментов программы построены оси проксимального и дистального фрагментов и скиаграммы. Темп коррекции был выбран 1 мм/сут. При этом при помощи оригинальных телескопических стоек, так называемых страт, узла Орто-СУВ коррекция выполнялась дробно 4 раза в день, следуя стандартам дистракции по

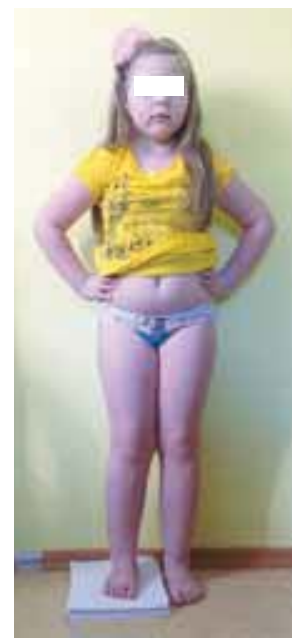

$a$

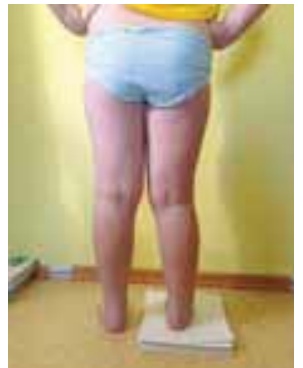

6

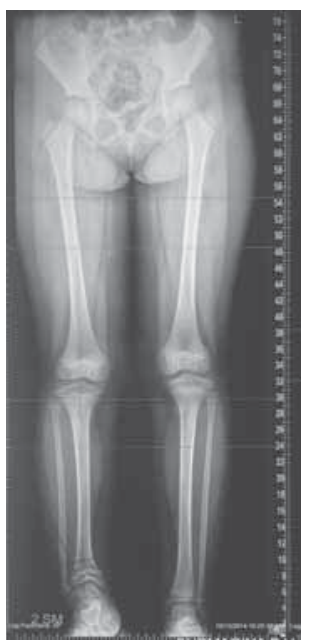

B

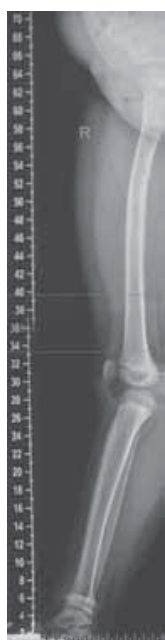

2

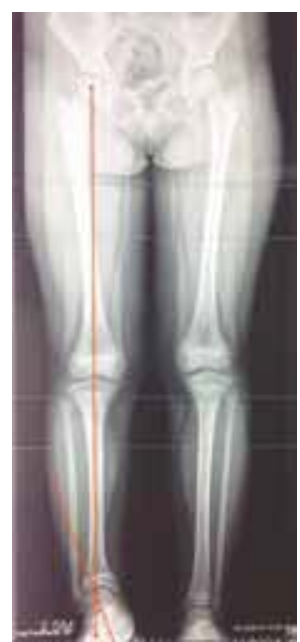

$\partial$

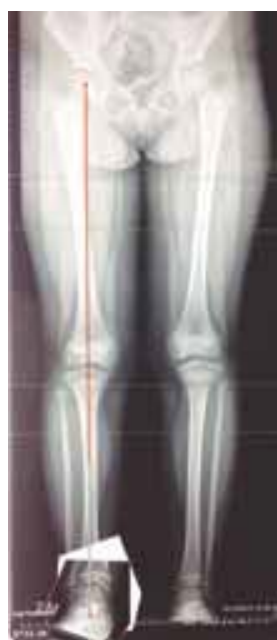

$e$

Рис. 1. Пациентка Б. до лечения: $a, 6-$ внешний вид; $8,2-$ телерентгенограммы нижних конечностей; $\partial-$ на рентгенограмме проведены механические оси проксимального и дистального костных фрагментов, найдена вершина и угол деформации; $e-$ в программе BoneNinja выполнена «имитирующая» остеотомия скиаграммы на уровне вершины, деформация устранена 


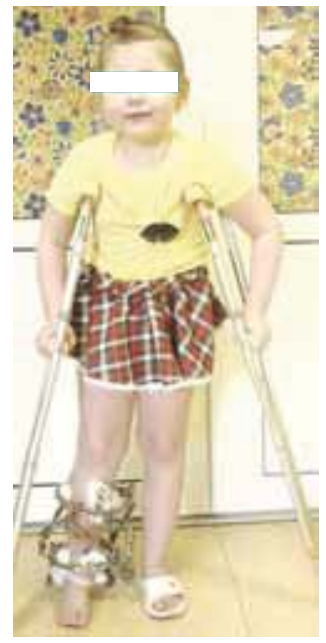

$a$

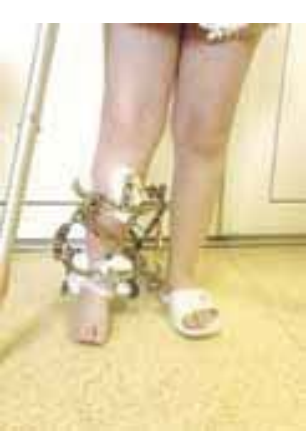

6
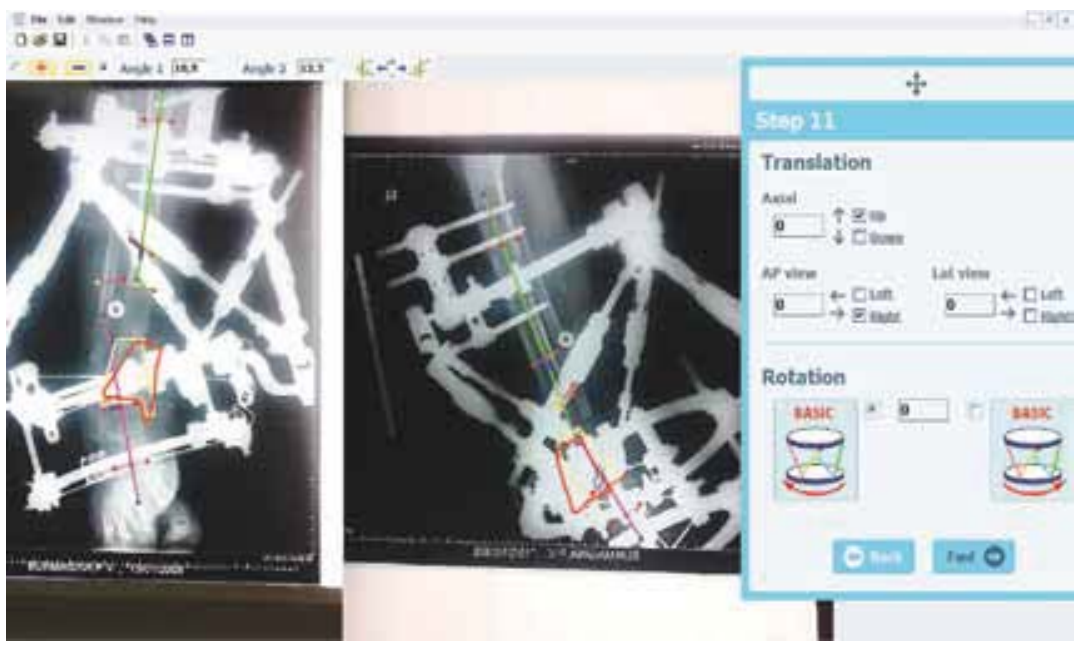

B

Рис. 2. Пациентка Б. после операции: $a, 6-$ внешний вид ребенка и конечности после операции; $в-$ расчет коррекции деформации в программе, прилагаемой к аппарату Орто-СУВ на шаге 11, где желтый контур - скиаграмма дистального костного фрагмента на момент расчета, красный контур - ожидаемое конечное положение дистального костного фрагмента после коррекции деформации

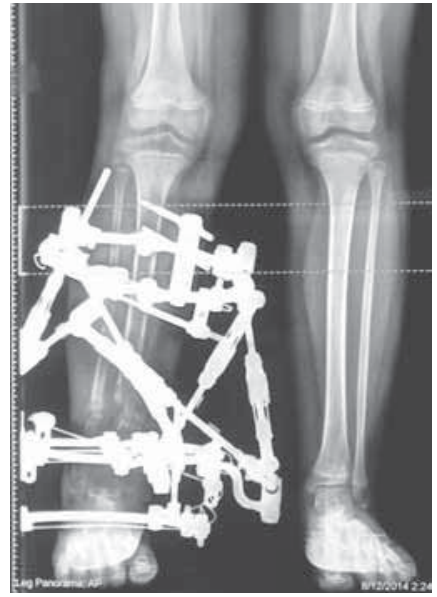

$a$

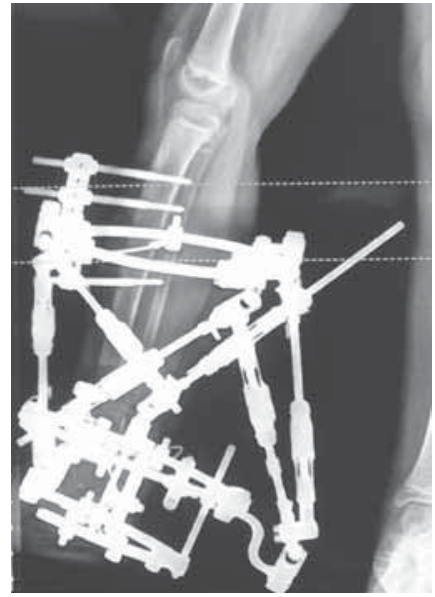

6

Рис. 3. Пациентка Б.: $a, 6$ - рентгенограммы после коррекции деформации

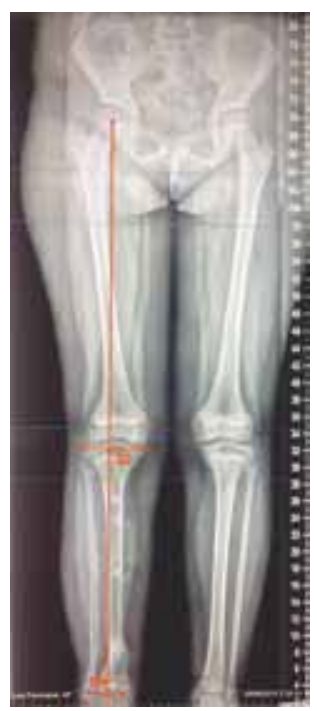

$a$

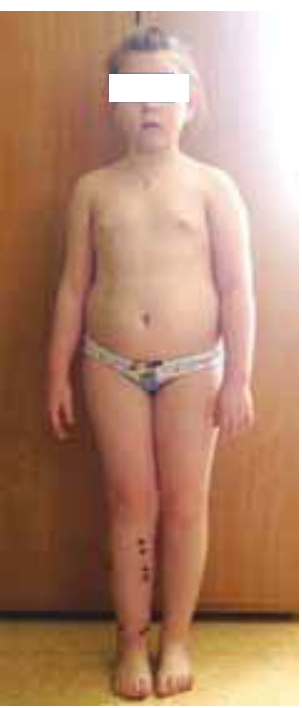

6

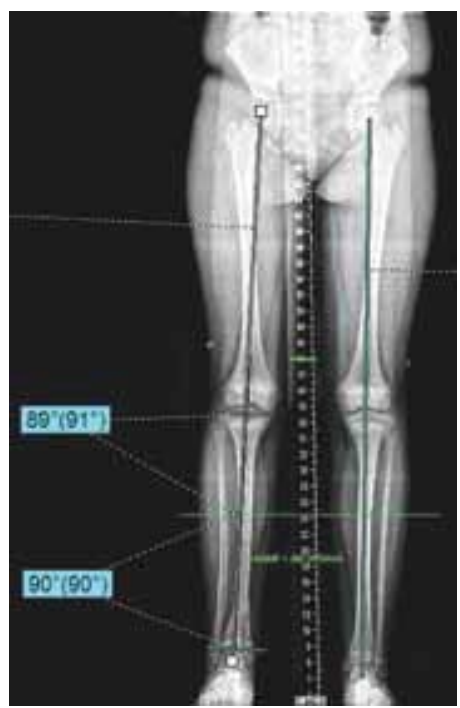

B

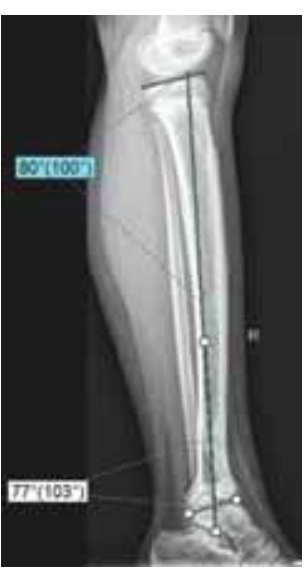

2

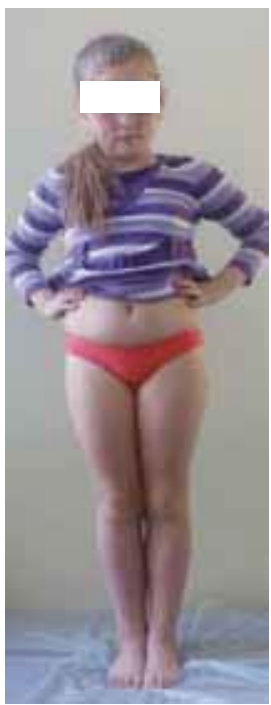

$\partial$

Рис. 4. Пациентка Б.: $a, 6-$ фотографии и панорамные рентгенограммы после демонтажа аппарата внешней фиксации; в, 2, $\partial$ - фотографии и рентгенограммы через 2 года после демонтажа КДА 
Илизарову. Период дистракции составил 46 дней, период коррекции деформации - 15 дней. Деформация была устранена (рис. $3, a, 6$ ). Совокупное удлинение (измеренное по панорамным рентгенограммам) составило 52 мм: величина удлинения 35 мм, величина «переудлинения» -17 мм. Через 1 месяц после окончания коррекции деформации демонтирована опора со стопы («разблокирован» голеностопный сустав). В дальнейшем осуществлялись ЛФК, механотерапия. Аппарат демонтирован через 198 дней после операции (рис. 4, $a, 6)$. Индекс внешней фиксации составил 26,3 дня/см. Достигнуты следующие параметры референтных линий и углов: проксимальный медиальный механический угол большеберцовой кости $-89^{\circ}$; дистальный латеральный механический угол большеберцовой кости $-89^{\circ}$; анатомический задний проксимальный большеберцовый угол $-80^{\circ}$; анатомический передний дистальный большеберцовый угол $-77^{\circ}$.

На контрольном осмотре через 2 года после демонтажа аппарата внешней фиксации (рис. 4 , в, $2, \partial$ ) длины конечности коррекция деформации сохраняется, конечности равной длины.

\section{ЗакАючение и выводы}

Использование методики эпифизиодеза неповрежденной порции зоны роста в сочетании с остеотомией и чрескостным остеосинтезом с последующими дозированными коррекцией деформации и удлинением достоверно снижает частоту рецидивов у пациентов с деформациями голени на фоне физарных синостозов. Применение чрескостных устройств на базе компьютерной навигации обеспечивает высокую точность коррекции деформации голени у детей.

\section{Информация о финансировании и конфликте интересов}

Автор В.А. Виленский декларирует то, что является сотрудником фирмы ООО «Орто-СУВ».

\section{Список митературы}

1. Seel EH, Noble S, Clarke NM, Uglow MG. Outcome of distal tibial physeal injuries. J Pediatr Orthop $B$. 2011;20(4):242-8. doi: 10.1097/BPB.0b013e3283467202.

2. Shaw N, Erickson C, Bryant $S$, et al. Regenerative Medicine Approaches for the Treatment of Pediatric Physeal Injuries. Tissue Eng Part B Rev. 2017Aug22. doi: 10.1089/ten.TEB.2017.0274. [Epub ahead of print] PubMed PMID: 28830302.

3. Лекишвили М.В. Изучение влияния одномоментной дозированной дистракции на восстановление поврежденной ростковой зоны длинных костей: автореф. дис. ... канд. мед. наук. - М., 1997. - 18 с. [Lekishvili MV. Izucheniye vliyaniya odnomomentnoy dozirovannoy distraktsii na vosstanovleniye povrezhdennoy rostkovoy zony dlinnykh kostey [dissertation]. Moscow; 1997. 18 p.]

4. Brouwer GM, Tol AW, Bergink AP, Belo JN, et al. Association between valgus and varus alignment and the development and progression of radiographic osteoarthritis of the knee. Arthritis Rheum. 2007;56(4):12041211. doi: 10.1002/art.22515.

5. Krause F, Veljkovic A, Schmid T. Supramalleolar Osteotomies for Posttraumatic Malalignment of the Distal Tibia. Foot Ankle Clin. 2016;21(1):1-14. doi: 10.1016/j. fcl.2015.09.001.

6. Saltzman CL, Salomon ML, Blanchard GM, et al. Epidemiology of ankle arthritis: report of a consequentive series of 639 patients from a tertiary orthopedic center. Iowa Orthop J. 2005;25:44-6.

7. Khoshhal KI, Kiefer GN. Physeal bridge resection. J Am Acad Orthop Surg. 2005;13(1):47-58. doi: 10.5435/00124635-200501000-00007.

8. Williamson RV, Staheli LT. Partial physeal growth arrest: treatment by bridge resection and fat interposition. J Pediatr Orthop. 1990;10(6):769-776. doi: 10.1097/01241398-199011000-00012.

9. Илизаров Г.А., Девятов А.А. Оперативное удлинение голени с одновременным устранением деформаций // Ортопедия, травматология и протезирование. - 1969. - № 3. - С. 32-37. [Ilizarov GA, Devyatov AA. operativnoye udlineniye goleni s odnovremennym ustraneniyem deformatsiy. Ortopediya, travmatologiya i protezirovaniye. 1969;(3):32-37. (In Russ.)]

10. Birch JG, Samchukov ML. Use of the Ilizarov method to correct lower limb deformities in children and adolescents. J Am Acad Orthop Surg. 2004;12(3):144-154. doi: 10.5435/00124635-200405000-00002.

11. Gubin AV, Borzunov DY, Malkova TA. The Ilizarov paradigm: thirty years with the Ilizarov method, current concerns and future research. Int Orthop. 2013;37:1533-1539. doi: 10.1007/s00264-013-1935-0.

12. Madhuri V, Gangadharan S. Acute Deformity Correction Using an Osteotomy in Pediatric Lower Limb Deformities (S. Sabharwal). 2016:79-103. doi: 10.1007/978-3-319-17097-8_6.

13. Horn J, Kristiansen LP, Steen H. Deformity correction during growth after partial physeal arrest. Acta Orthop Belg. 2009;75(2):219-24.

14. Lee SC, Shim JS, Seo SW, et al. The accuracy of current methods in determining the timing of epiphysiodesis. Bone Joint J. 2013;95-B(7):993-1000. doi: 10.1302/0301-620X.95B7.30803.

15. Horn J, Steen H, Huhnstock S, et al. Limb lengthening and deformity correction of congenital and acquired deformities in children using the Taylor Spatial Frame. Acta Orthopaedica. 2016;88(3):334-340. doi: 10.1080/17453674.2017.1295706.

16. Tsibidakis H, Kanellopoulos AD, Sakellariou VI, et al. The role of Taylor Spatial Frame for the treatment of 
acquired and congenital tibial deformities in children. Acta Orthop Belg. 2014;80:419-25.

17. Keshet D, Eidelman M. Clinical utility of the Taylor spatial frame for limb deformities. Orthopedic Research and Reviews. 2017;9:51-61. doi: 10.2147/orr.s113420.

18. Paley D. History and Science Behind the Six-Axis Correction External Fixation Devices in Orthopaedic Surgery. Oper Tech Orthop. 2011;21(2):125-128. doi: 10.1053/j.oto.2011.01.011.

19. Соломин Л.Н. Основы чрескостного остеосинтеза. Частные вопросы - 2. - 2-е изд. - М.: Изд-во БИНОМ, 2015. - Т. 2. - 560 с. [Solomin LN. Osnovy chreskostnogo osteosinteza. Chastnyye voprosy -2 . Moscow: Izd-vo BINOM; 2015. Vol. 2. 560 p. (In Russ.)]

20. Соломин Л.Н., Виленский В.А. Практическая классификация деформаций длинных трубчатых костей // Травматология и ортопедия России. 2008. - № 3 (Приложение). - С. 44. [Solomin LN, Vilenskij VA. Prakticheskaja klassifikacija deformacij dlinnyh trubchatyh kostej. Travmatologija i ortopedija Rossii. 2008(3)(Appl):44. (In Russ.)]

21. Bright RW. Partial growth arrest: identification, classification, and results of treatment. Orthop Trans. 1982;6:65-66.

22. Loder RT, Johnston CE II. Infantile tibia vara. J Pediatr Orthop. 1987;7(6):639-646. doi: 10.1097/01241398198707060-00002.

23. Chotigavanichaya C, Salinas G, Green T, et al. Recurrence of varus deformity after proximal tibial osteotomy in Blount disease: long-term follow-up. J Pediatr Orthop. 2002;22(5):638-41. doi: 10.1097/01241398200209000-00013.

24. Домингуш М. Повреждения зон роста длинных костей у детей и их лечение: автореф. дис. ... канд. мед. наук. - M., 1997. - 18 с. [Domingush M. Povrezhdeniya zon rosta dlinnykh kostey u detey i ikh lecheniye. [dissertation]. Moscow; 1997. 18 p. (In Russ.)]

25. Sabharwal S. Blount's disease, in Limb lengthening and reconstruction surgery. Informa healthcare. New YorkLondon; 2007. P. 511-520.
26. Соломин Л.Н., Щепкина Е.А., Виленский В.А., и др. Коррекция деформаций бедренной кости по Илизарову и основанным на компьютерной навигации аппаратом «Орто-СУВ» // Травматология и ортопедия России. - 2011. - № 3. - С. 32-39. [Solomin LN, Shhepkina EA, Vilenskij VA, et al. Korrekcija deformacij bedrennoj kosti po Ilizarovu i osnovannym na komp'juternoj navigacii apparatom Orto-SUV. Travmatologija i ortopedija Rossii. 2011;(3):32-39. (In Russ.)]

27. Виленский В.А., Поздеев А.А., Зубаиров Т.Ф., и др. Лечение детей с деформациями длинных трубчатых костей нижних конечностей методом чрескостного остеосинтеза с использованием аппарата Орто-СУВ: анализ 213 случаев // Ортопедия, травматология и восстановительная хирургия детского возраста. - 2016. - № 4. - С. 21-32. doi: 10.17816/ PTORS4421-32 [Vilenskij VA, Pozdeev AA, Zubairov $\mathrm{TF}$, et al. Lechenie detej s deformacijami dlinnyh trubchatyh kostej nizhnih konechnostej metodom chreskostnogo osteosinteza s ispol'zovaniem apparata Orto-SUV: analiz 213 sluchaev. Ortopedija, travmatologija $i$ vosstanovitel'naja hirurgija detskogo vozrasta. 2016;(4):21-32. (In Russ.)]. doi: 10.17816/ PTORS4421-32.

28. Westh RN, Menelaus MB. A simple calculation for the timing of epiphyseal arrest: a further report. J Bone Joint Surg. 1981;63B:117-119.

29. Moseley CF. A straight-line graph for leg-length discrepancies. J Bone Joint Surg. 1977;59(2):174-179. doi: 10.2106/00004623-197759020-00006.

30. Anderson M, Green WT, Messner MB. Growth and predictions of growth in the lower extremities. $J$ Bone Joint Surg. 1963;45(1):1-14. doi: 10.2106/00004623196345010-00001.

31. Paley D, Bhave A, Herzenberg JE, Bowen JR. Multiplier method for predicting limb-length discrepancy. J Bone Joint Surg. 2000;82(10):1432-1446. doi: 10.2106/00004623-200010000-00010.

32. Aguilar JA, Paley D, Paley J, et al. Clinical validation of the multiplier methodfor predicting limb length discrepancy and outcome at epiphysiodesis: part II. J Pediatr Orthop. 2005;25(2):192-196. doi: 10.1097/01. bpo.0000150808.90052.7c.

\section{Сведения об авторах}

Виктор Александрович Виленский - канд. мед. наук, старший научный сотрудник отделения костной патологии ФГБУ «НИДОИ им. Г.И. Турнера» Минздрава России, Санкт-Петербург. E-mail: vavilensky@mail.ru.

Андрей Александрович Поздеев - канд. мед. наук, врач травматолог-ортопед отделения костной патологии ФГБУ «НИДОИ им. Г.И. Турнера» Минздрава России, Санкт-Петербург. E-mail: aapozdeev@gmail.com.

Тимур Фаизович Зубаиров - канд. мед. наук, научный сотрудник отделения костной патологии ФГБУ «НИДОИ им. Г.И. Турнера» Минздрава России, СанктПетербург.

Екатерина Анатольевна Захарьян - канд. мед. наук, научный сотрудник отделения костной патологии ФГБУ «НИДОИ им. Г.И. Турнера» Минздрава России, Санкт-Петербург. E-mail: zax-2008@mail.ru.
Viktor A. Vilensky - MD, $\mathrm{PhD}$, senior research associate of the Department of Bone Pathology. The Turner Scientific Research Institute for Children's Orthopedics, Saint Petersburg, Russia. E-mail: vavilensky@mail.ru.

Andrey A. Pozdeev - MD, $\mathrm{PhD}$, orthopedic and trauma surgeon of the Department of Bone Pathology. The Turner Scientific Research Institute for Children's Orthopedics, Saint Petersburg, Russia. E-mail: aapozdeev@gmail.com.

Timur F. Zubairov - MD, PhD, research associate of the Department of Bone Pathology. The Turner Scientific Research Institute for Children's Orthopedics, Saint Petersburg, Russia.

Ekaterina A. Zakharyan - $\mathrm{MD}, \mathrm{PhD}$, research associate of the Department of Bone Pathology. The Turner Scientific Research Institute for Children's Orthopedics, Saint Petersburg, Russia. E-mail: zax-2008@mail.ru. 